Article

\title{
Applying Artificial Intelligence to Improve On-Site Non-Destructive Concrete Compressive Strength Tests
}

\author{
Tu Quynh Loan Ngo ${ }^{1}$ (D), Yu-Ren Wang ${ }^{2, *(\mathbb{D})}$ and Dai-Lun Chiang ${ }^{2}$ \\ 1 Faculty of Industrial Fine Arts, Ton Duc Thang University, Ho Chi Minh City 758307, Vietnam; \\ ngotuquynhloan@tdtu.edu.vn \\ 2 Department of Civil Engineering, National Kaohsiung University of Science and Technology, \\ Kaohsiung City 80778, Taiwan; 104312137@gm.kuas.edu.tw \\ * Correspondence: yrwang@nkust.edu.tw
}

check for

updates

Citation: Ngo, T.Q.L.; Wang, Y.-R.; Chiang, D.-L. Applying Artificial Intelligence to Improve On-Site

Non-Destructive Concrete

Compressive Strength Tests. Crystals 2021, 11, 1157. https://doi.org/ $10.3390 /$ cryst11101157

Academic Editors: Weiqiang Wang, Beatrice Pomaro, Yang Yu and Rafael Shehu

Received: 30 June 2021

Accepted: 9 September 2021

Published: 23 September 2021

Publisher's Note: MDPI stays neutral with regard to jurisdictional claims in published maps and institutional affiliations.

Copyright: (c) 2021 by the authors. Licensee MDPI, Basel, Switzerland. This article is an open access article distributed under the terms and conditions of the Creative Commons Attribution (CC BY) license (https:// creativecommons.org/licenses/by/ $4.0 /)$.

\begin{abstract}
In the construction industry, non-destructive testing (NDT) methods are often used in the field to inspect the compressive strength of concrete. NDT methods do not cause damage to the existing structure and are relatively economical. Two popular NDT methods are the rebound hammer (RH) test and the ultrasonic pulse velocity (UPV) test. One major drawback of the RH test and UPV test is that the concrete compressive strength estimations are not very accurate when comparing them to the results obtained from the destructive tests. To improve concrete strength estimation, the researchers applied artificial intelligence prediction models to explore the relationships between the input values (results from the two NDT tests) and the output values (concrete strength). In-situ NDT data from a total of 98 samples were collected in collaboration with a material testing laboratory and the Professional Civil Engineer Association. In-situ NDT data were used to develop and validate the prediction models (both traditional statistical models and AI models). The analysis results showed that AI prediction models provide more accurate estimations when compared to statistical regression models. The research results show significant improvement when AI techniques (ANNs, SVM and ANFIS) are applied to estimate concrete compressive strength in RH and UPV tests.
\end{abstract}

Keywords: adaptive neural fuzzy inference system; artificial intelligence; support vector machine; artificial neural network; concrete strength; non-destructive testing; rebound hammer test; ultrasonic pulse velocity

\section{Introduction}

Nowadays, artificial intelligence (AI) methods such as artificial neural networks (ANNs), support vector machines (SVM) and adaptive neural fuzzy inference systems (ANFIS) are commonly applied in various research fields. Several research results have shown that ANNs, SVM and ANFIS are effective AI prediction methods [1-5]. In the construction industry, non-destructive testing (NDT) methods are used to examine the compressive strength of concrete because they are important alternatives to destructive tests, and in the meantime, are relatively easy to conduct and are economical. If destructive methods are used in the lab, the resulting compressive strength test results would not accurately represent the quality of the in-situ cast concrete. This is because the strength and quality of the in-situ concrete might be affected by many factors such as transportation, placement, tamping and curing. Testing core samples from an existing structure is a better way to examine the concrete quality. However, it is sometimes not feasible to take core samples on site. Oftentimes, the drilled samples or the concrete structure might be damaged during the drilling process. Therefore, NDT are good alternatives to measure the compressive strength of concrete. Rebound Hammer (RH) and Ultrasonic Pulse Velocity (UPV) tests are two popular NDT methods that can be used to estimate the compressive strength of concrete. Without damaging the structure, they can effectively evaluate the uniformity and relative quality of concrete structures. The RH test, however, provides 
estimated results with a large percentage of errors compared to the results obtained from using destructive testing methods, and for UPV testing method, the estimated results are not quite accurate. In addition, the actual compressive strength of concrete is unconfined and self-compacted [6]. This research adopts AI techniques to analyze Silver Schmidt $\mathrm{RH}$ and UPV test results. The goal of this research is to develop more accurate concrete compressive strength prediction models.

The RH test is relatively simple to conduct. First, the plunger of a rebound hammer is pressed against the surface of a concrete sample; then, a graduated scale is used to measure the mass rebound; finally, the rebound value is read in terms of the rebound number or rebound index. The compressive strength is calculated using the conversion chart provided by the manufacturer $[7,8]$. The UPV tests are based on the principles of measuring the propagation time of an ultrasonic pulse wave in concrete samples. After the velocity is calculated, the compressive strength is then evaluated. It has been shown in previous studies that the higher the velocity of an ultrasonic pulse wave, the better the quality of a concrete sample is $[9,10]$. To predict the compressive strength of concrete, a prediction model needs to be constructed. In addition to the traditional statistical prediction model, artificial intelligence prediction models are also adopted to evaluate the property of concrete sample [11,12].

This investigation incorporates ANNs, SVMs and ANFIS to develop prediction models. The estimation results show significant improvement when compared to traditional estimation methods. A total of 98 in-situ test samples were collected for study. Among them, 70 were randomly chosen to be the training dataset and the remaining 28 samples were used for model validation. Both traditional statistical models and AI models were developed and tested using the sample data. Analysis results showed that the AI models could provide better concrete compressive strength prediction results.

\section{Research Significance}

Most of the previous research testing the compressive strength of concrete has used lab concrete samples for the NDT, while this research uses drilled samples from existing structures. Both artificial intelligence along with traditional statistical analysis methods are employed to investigate the relationship between the compressive strength of the concrete samples and the SONREB (UPV + RH) test results. For future research, more samples from other existing buildings could be collected for further analysis. In addition, other non-destructive methods can be incorporated for further investigation.

The remainder of this paper is organized as follows: Section 3 introduces related work applying AI in combination with NDT; Section 4 describes the experiment methodology; The research results are presented in Section 5. Then, the conclusions are made in Section 6.

\section{Literature Review}

In assessing concrete structure, destructive methods are sometimes not preferred because they might cause damage to the concrete. This makes the NDT method a popular alternative. NDT methods do not require a lot on sample preparation, and the test equipment is relatively simple [13]. Due to laboratory equipment limitations, NDT methods are preferable for the prediction of concrete compressive strength [14]. Based on the relationship between material strength and NDT parameters, the concrete strength of concrete will be evaluated. Detailed information regarding the empirical relationship between the concrete strength and the NDT parameters is provided by the NDT equipment manufacturers. Hence, calibration is necessary for different types of concrete $[15,16]$. RH and UPV testing methods are among the most popular NDT methods that are successfully applied to evaluate the properties of concrete.

The RH test is one of the most popular NDT methods to measure the compressive strength of concrete. The process of the RH test is summarized as follows [17]: an elastic mass will strike the surface of concrete sample, and its rebound depends on the hardness of the concrete sample. First the plunger should be pressed against the test surface, the 
weight will then strike the surface and the mass rebound is measured from a graduated scale. The rebound index (or rebound number) is read from the test hammer. In the RH test, the rebound number is one of the concrete strength indicators. The rebound number is measured through the energy that was absorbed during the impact [18]. In addition, there are also other factors that affect the rebound number, such as the moisture content of the concrete, the surface smoothness, the nature of the coarse aggregate, the age of the concrete, the size and shape of the concrete, and the rigidity of the concrete specimen [19]. Although there has not been a universal relationship developed between concrete strength and surface hardness, several researchers have tried to examine the relationship for a given concrete [20]. The detailed procedure of the RH experimental setup in this research follows ASTM C805 [21].

In the UPV test, ultrasonic pulse wave velocity is measured in order to estimate the material strength. This technique adopts the theory of wave propagation in order to measure the depth of the material and to determine the internal cracks or damage [22-24]. The ultrasonic pulse velocity of the concrete is affected by age and is inversely proportional to the volume of pores in the concrete [25]. The rate of UPV change in concrete is used to determine the setting of the concrete and to specify the different stages of change at early ages at the microstructural level $[26,27]$. Some researchers also state that UPV is exaggerated by the variation at the microstructural level in the mortar and is used to estimate the sand content in the mortar [28]. To detect damage inside the concrete, the UPV is also used in some research $[29,30]$. For other types of concrete, such as lightweight or asphalt concrete, UPV tests were also conducted to evaluate the properties of samples $[31,32]$. UPV measures the ultrasonic pulse wave velocity when it penetrates through materials. The measurement is used to predict the strength of the materials, find any changes in the condition of the materials, internal flaws, and other factors. The UPV technique, however, is not always practical for all type of concrete samples. Because sound waves are used in UPV tests, the test is not accurate for samples that have internal water-filled cracks and rough surfaces [33]. To increase the accuracy of the UPV tests, coupling gels are applied in between the transducer of the testing equipment and roughness samples to smooth the contact layer. This ultrasonic pulse velocity is affected by various factors, such as the aggregate, water/cement ratio, moisture, and so on. Previous research has used UPV test results to evaluate the compressive strength, modulus of elasticity, aggregate type, and moisture content of the concrete, etc. [34]. A detailed description of UPV test as well as a detailed description of the correlation between the compressive strength, the pulse velocity, and the elastic modulus can be found in ASTM C597-83 [35]. The ultrasonic pulse velocity is determined by the density and elasticity of the material sample and considers factors such as the cement type, the concrete mix, the aggregate type, the permeability, porosity, density, cement hydration, curing, water cement ratio, and the manufacturing process [36-38]. This complexity makes the ultrasonic waves irregular and affects the concrete compressive strength estimations in non-destructive tests [39]. The aggregate size affects the ultrasonic pulse velocity, as a larger aggregate results in a higher pulse velocity [40]. In addition, the production process also affects the behavior of UPV. For instance, prolonging the curing period amplifies the UPV values. A longer curing period increases the UPV because the ratio of gel/space changes during a longer curing period [41,42]. The UPV value decreases when the oxygen permeability and porosity of the sample material is increased, which indicates that an ultrasonic pulse travels faster in solids than in a void [43]. Islam et al. [44] showed the effect of peat presence on cement-sand-bricks. A relationship between the UPV and the wet compressive strength of the peat-enhanced bricks was found. Hence, reducing the compressive strength would lead to the UPV values to drop for cementation products.

Upon applying AI in predicting concrete strength, most research work focusses on applying neural networks for the prediction. Some of the latest works discussing the use of ANNs are briefly summarized in this section. Asteris and Mokos applied ANNs to predict the compressive strength of concrete. They proposed two ANNs models that can be reliably 
applied for data obtained by NDT tests with some constraints on the data value [45]. Using a hybrid AI method, Gholamreza and Arash studied the radial basic function of neural networks in combination with the firefly algorithm to predict the compressive strength of concrete [46]. Bonagura and Bobile verified the accuracy of the artificial neural approach in predicting the compressive strength compared to parametric multi-variable regression models [47]. It is noted that these research works used the database obtained from previous studies. For this study, the researchers collaborated with a material testing laboratory and the Professional Civil Engineer Association to obtain the experimental data for the in-situ concrete samples. In addition to ANNs, other AI methods such as SVMs and ANFIS were also adopted to develop and validate prediction models for concrete compressive strength.

\section{Experiment Methodology}

The data collection was done in collaboration with a material testing laboratory in Taiwan. The authors of this paper have signed a non-disclosure agreement to keep the raw data information confidential. Hence, only limited data can be revealed to the public. Data from a total of 98 samples were collected for this research. The descriptive statistics of the data are shown in Table 1. The scatter plots of the actual strength of the concrete samples (obtained from destructive tests) and the NDT test results (RH and UPV) are shown in Figure 1.

Table 1. Summary of concrete sample test results.

\begin{tabular}{ccccc}
\hline Test Results & Max & Min & Average & Stdev. \\
\hline Rebound value $(n=98 \times 10)$ & 59.400 & 36.650 & 50.698 & 4.516 \\
\hline Average wave speed $(\mathrm{UPV})(\mathrm{m} / \mathrm{s})(n=98)$ & 155.600 & 111.475 & 125.588 & 8.0613 \\
\hline Actual compressive strength $(\mathrm{MPa})(n=98)$ & 65.998 & 15.495 & 47.759 & 9.660 \\
\hline
\end{tabular}
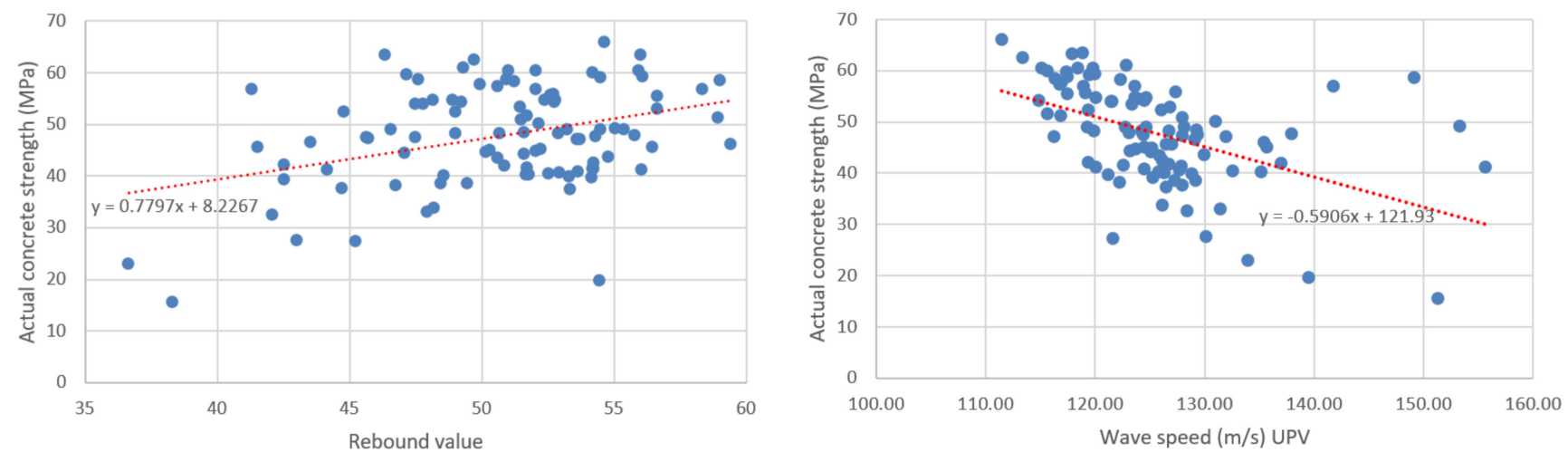

Figure 1. Actual concrete strength linear fit scatterplot.

Three AI techniques (ANNs, SVM and ANFIS) were used to develop the prediction model in this study. The performance of the model to predict concrete compressive strength was expected to improve due to the use of the AI approach, especially when compared to conventional approaches. The model development process is shown in Figure 2. According to Khademi [48], the generalization aibility of the predictive models can be enhanced, but their effectiveness needs further examination. 


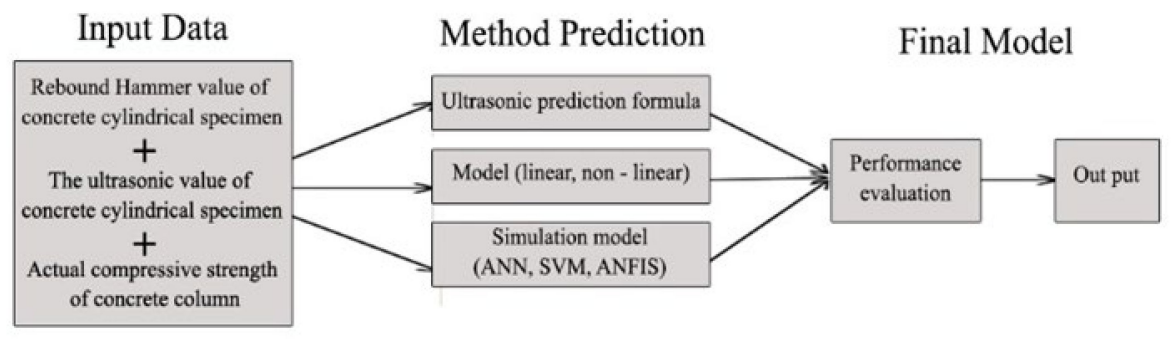

Figure 2. Model development.

\subsection{Artificial Intelligence Prediction Methods}

ANNs, SVM, and ANFIS are used to develop the concrete compressive strength prediction models for the RH and UPV tests. These models are designed and applied to arbitrate non-linear problems. To search for more accurate prediction results, this research uses these three methods to develop prediction models to determine concrete compressive strength.

\subsubsection{Overview of Artificial Neural Networks}

Artificial neural networks simulate the human brain in terms of two important features: learn and adapt. ANNs have been applied in many areas of engineering-related fields, such as pattern recognition, system model and control, system identification, and classification tasks [49]. Previous research results show that ANNs outperform traditional methods such as multiple regression analysis or multivariate analysis [50]. In ANNs, a highly interconnected system with simple processing elements has the ability to learn the complex interrelationship between independent and dependent variables [51]. A neuron is defined as an information processing system unit that consists of a connecting link, which is a summation with/without bias and an activation function [52]. Each neuron, as shown in Figure 3, receives inputs and weights from neurons in previous layer using mathematical training processes.

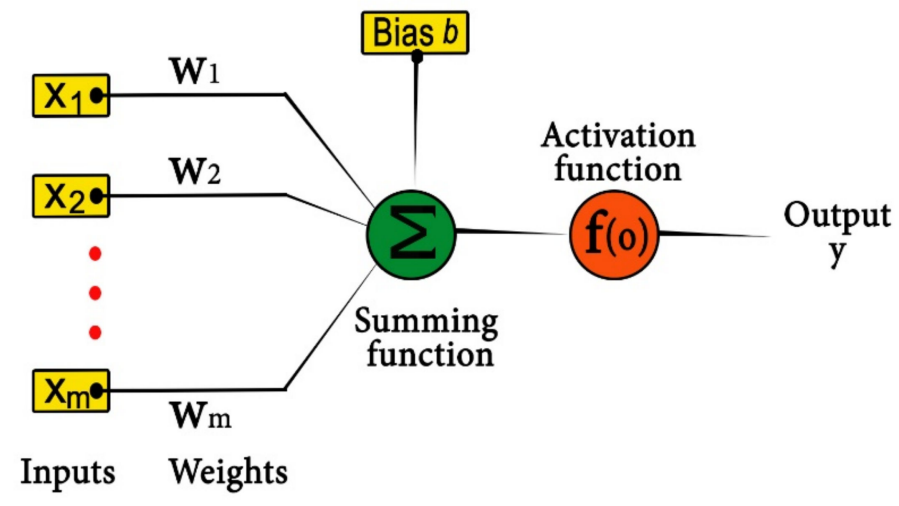

Figure 3. Model of network block.

The weighted sum of input data is used as an argument for an activation function to form an output [53]. The output of a neuron is calculated follow Equations (1)-(3):

$$
\begin{gathered}
u=\sum_{j=1}^{m} w_{j} x_{j}+b \\
y=f(u) \\
f(u)=\frac{1+e^{-u}}{1-e^{-u}}
\end{gathered}
$$


where $x_{j}(j=1, \ldots, m)$ is the input signal from previous layer; $w_{j}(j=1, \ldots, m)$ is the weight associated with $x_{j} ; m$ is the number of inputs; $b$ is the bias; and $f(u)$ is the activation function. The activation function takes the form of sigmoid functions (logistic) or linear function as given in Equations (4) and (5).

Logistic transfer function (logsig):

$$
f(u)=\frac{1}{1+e^{-u}}
$$

Linear transfer function (pure line):

$$
f(u)=u
$$

\subsubsection{Multilayer-Feedforward Neural Network}

For multi-layer ANNs, neurons in the networks are arranged in layers as shown in Figure 4. In a multilayered feed-forward neural network (FFNN), the outputs of the previous layer become the inputs of the following layer. The layers between the input layer and output layer are hidden layers. More hidden layers indicate the higher complexity of the approximation function of the network. One or two hidden layers are capable of approximating an arbitrarily complex mapping within a finite dataset of patterns [54].

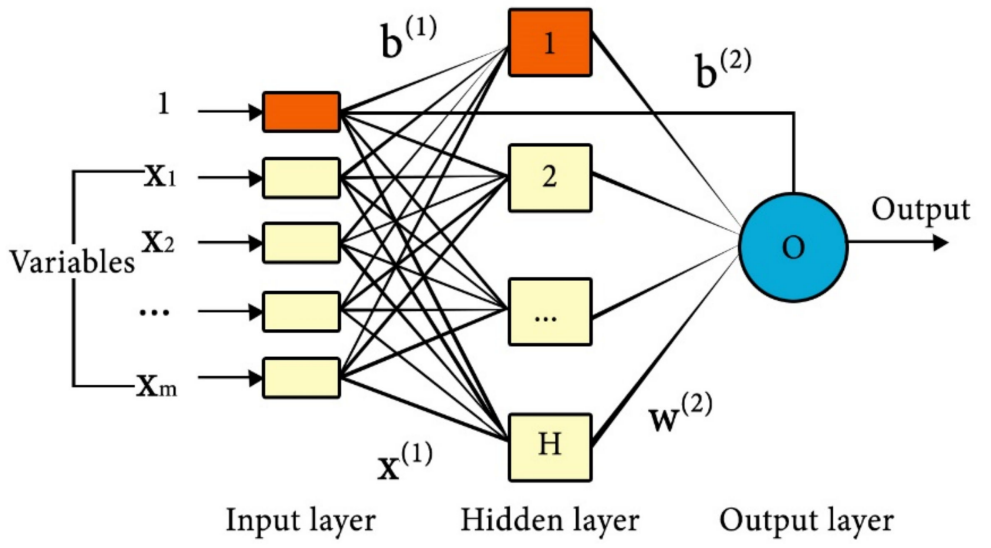

Figure 4. A typical architecture of a multilayer neural network.

\subsubsection{Radial Basis Function Neural Network}

An alternative multilayered feed-forward neural network is the radial basis function neuron network (RBFNN), which was proposed by Samarasinghe [55]. An RBFNN has three layers: the input layer, a single hidden layer, and the output layer. Compared to FFNN, RBFNN has some advantages, such as its high training speed and the fact that it is less vulnerable to problems with non-stationary inputs [56]. The hidden neurons distinguish the two types of neural networks. FFNN uses the S-shaped sigmoid activation function while RBFNN uses the Gaussian radial basis function. In RBFNN, there are vector connecting weights, w, between the output layer and the hidden layer. However, there are no weights between the input layer and the hidden layer. Figure 5 illustrates an RBFNN with a single output. 


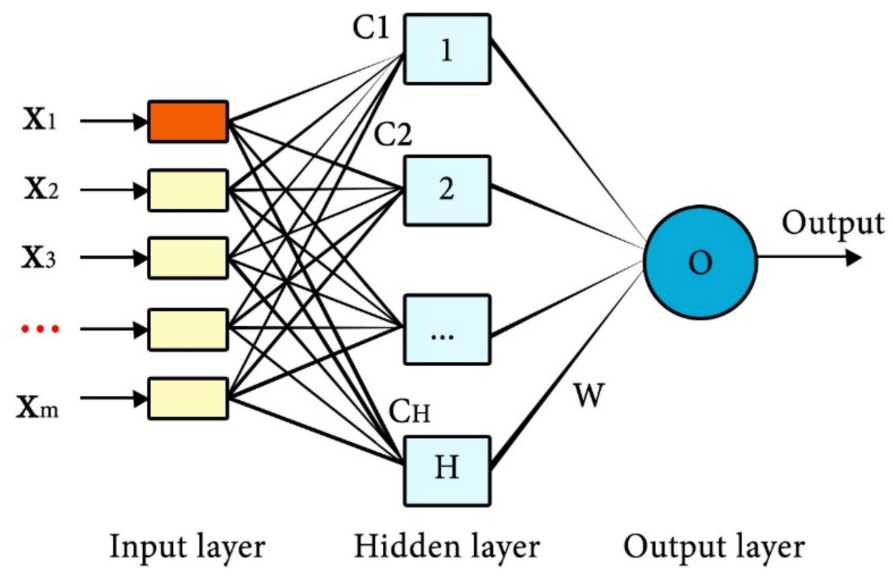

Figure 5. A typical architecture of an RBF neural network.

\subsubsection{General Linear Regression}

Estimating the correlation between inputs and outputs, general linear regression (GLR) determines how a dependent variable, which is a response variable, reacts to the independent variables, which are predictors. GLR is categorized into linear and non-linear regression. In linear regression, the margin of the training data input is maximized using the best hyperplane. The hyperplane boundary is defined as the training sample that has the shortest distance in the range of $\{-1,+1\}$ to the hyperplane. In non-linear regression, the data are transformed into a high dimension feature space using a mapping function $\Phi$.

Given that $X$ is the predictor variable and $y$ is the response variable, the relationship between $X$ and $y$ is estimated in GLR by forming a model using a link function, which is shown in Equation (6) [57].

$$
g(E(y))=X \times \beta+O, y \sim F
$$

where $g()$ is the selected link function, $O$ is the offset variable, $F$ is the distribution model of response variable $y, X$ is the predictor, and $\beta$ is the regression coefficient.

\subsubsection{Overview of Support Vector Machines}

Support vector machines (SVMs) are mechanical algorithms developed based on the theory of statistics. SVMs are usually suitable for small scale, nonlinear or high dimensional problems. This research uses SVMs to develop the prediction model for concrete compressive strength, using the experiment results from RH and UPV tests. The model prediction results are then compared with the real compressive strength. In SVMs, the upper boundary of the generalization error has been minimized. This enables the SVMs ability to process data better than other artificial intelligence techniques even for unseen data [58-60]. Some basic concepts of SVM models are described as follows [61]:

A dataset $G=\left\{\left(x_{u}, d_{i}\right)\right\}_{i}^{n}$ is given with input vector $x_{u}$ and target value $d_{i}$, and the size of the dataset is $n$. By mapping $\mathrm{x}$ into the high-dimensional feature space, $f(x)=\omega \Phi(x)+b$ is the non-linear regression function, where $\omega, \Phi$ and $b$ are the weight vector, the high-dimensional feature space, and the bias of the hyper plane, respectively. SVMs minimize the structural risks; therefore, the weight vector and the bias of the hyper plane can be obtained by minimizing the risk penalty function.

\subsubsection{Least Squares Support Vector Machines}

Least squares support vector machines (LS-SVMs) are used to solve linear systems instead of quadratic programming. These linear systems are known as the KarushKuhn-Tucker systems and can be used to investigate the numerical stability of these systems $[62,63]$. The conjugate gradient is one of the iterative methods that can be used to effectively solve these linear systems [64]. 
Let $\left\{\left(y_{k}, x_{k}\right)\right\}_{k=1}^{N}$, where $x_{k} \in R^{n}$ is the $k$ th input pattern and $y_{k} \in R$ is the $k$ th output pattern, be the training set with $\mathrm{N}$ data points. The support vector method is in the form of $y(x)=\operatorname{sign}\left[\sum_{k=1}^{N} \alpha_{k} y_{k} K\left(x, x_{k}\right)+b\right]$, with support values $\alpha_{k}, a$ constant $b$, and the kernel $K()$, where $K\left(x, x_{k}\right)=x_{k}^{T} x$ for linear SVMs; $K\left(x, x_{k}\right)=\left(x_{k}^{T} x+1\right)^{d}$ for the polynomial SVM of degree $\mathrm{d} ; K\left(x, x_{k}\right)=\tanh \left(K x_{k}^{T} x+\theta\right)$ for multilayer perceptron SVMs; and $K\left(x, x_{k}\right)=\exp \left(-\left\|x-x_{k}\right\| \frac{2}{2} / \sigma^{2}\right)$ for radial basic function SVMs.

\subsubsection{Overview of Adaptive Neuro Fuzzy Inference System}

For complex systems, ANFIS is widely used with the adaptation of the supervised learning algorithm. ANFIS is applied successfully when modeling non-linear functions, controlling the parameters of the induction machine, and predicting the chaotic time series. ANFIS has a structure that incorporates learning algorithms, fuzzy rules and multilayeredfeed forward networks, as shown in Figure 6 [65].

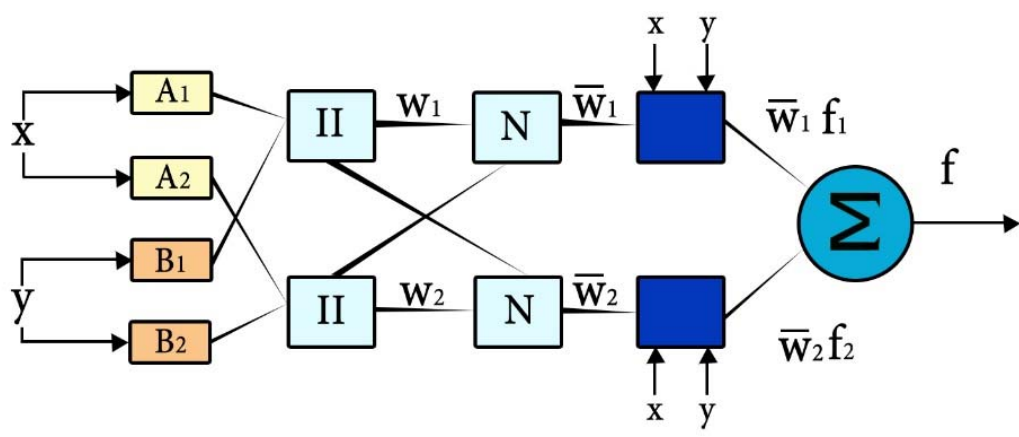

Figure 6. Structure of an ANFIS network.

By fixing the premise parameters, the overall output becomes a linear combination of the consequent parameters, and it is written as:

$$
f=\left(\bar{w}_{1} x\right) c_{11}+\left(\bar{w}_{1} y\right) c_{12}+\left(\bar{w}_{1}\right) c_{10}+\left(\bar{w}_{2} x\right) c_{21}+\left(\bar{w}_{2} y\right) c_{22}+\left(\bar{w}_{2}\right) c_{20}
$$

where $c_{i j}$ denotes the consequent parameters. The consequent parameters are adjusted by a hybrid algorithm in both forward and backward processes. In the forward process, the network inputs propagate forward until layer 4 , where the consequent parameters are identified by the least-squares method. In the backward process, the error signals propagate backward, and the premise parameters are updated by the gradient descent. Due to the decoupling of hybrid learning rule during the update for the premise and the consequent parameters, a shorter computational time is possible with the gradient method [65].

\subsubsection{ANFIS Modeling in MATLAB}

To find parameters that best fit the input data, the "ANFIS" function in the MATLAB Fuzzy Control Toolbox is used for prediction model development. This function provides an optimization scheme for the fuzzy system. The optimization can be completed with a neural network that does not require the computation of the gradient. When the initial condition of the fuzzy system is not clear and when there is no information on the type and number of membership functions, researchers have suggested the "genfis1" function for the initial modeling. This function will go through the data and find a good initial starting system $[65,66]$.

\subsection{In-Situ NDT Test and Lab Destructive Test}

In this study, the researchers collaborated with a professional government-certified material testing laboratory. This laboratory specializes in conducting destructive and nondestructive tests on construction materials. The NDT (RH and UPV) tests were conducted 
on 98 non-structure beams in the basement of a large residential complex. The beams were $50 \mathrm{~cm}$ in width and $70 \mathrm{~cm}$ in depth. For each beam, 10 rebound hammer measurements were taken with the Silver Schmidt N-Type electronic rebound hammer manufactured by PROCEQ. For the UPV tests, the test procedures followed method described in the study of Tharmaratnam and Tan [67] and the ASTMC59716: "the standard test method for pulse velocity through concrete". The instrument used for UPV tests was a TICO concrete ultrasonic detector developed by PROCEQ Company in Zurich, Switzerland. Four ultrasonic pulse velocity measurements were taken at each test location. After the $\mathrm{RH}$ and UPV tests, the core samples were taken at the same location. For consistency, all the RH and UPV tests were conducted by the same person and all the core samples were taken by another specialized individual. The collected data were used to develop and test the AI models. The in-situ data collection process is shown in Figure 7.

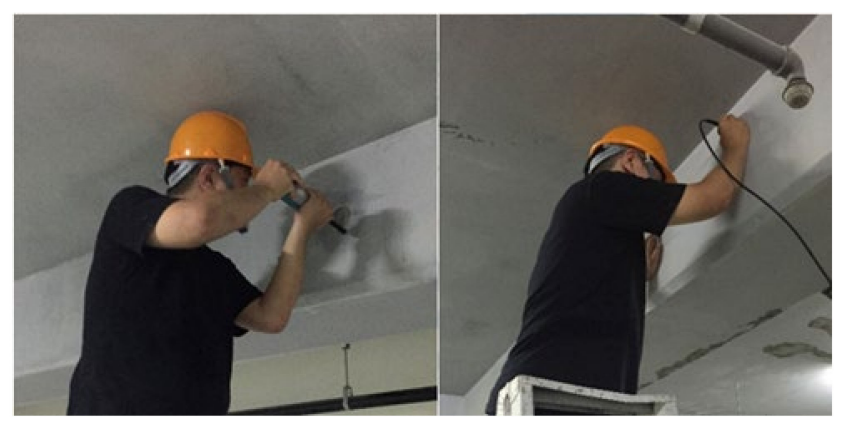

Figure 7. In-situ sample collection.

In this study, the actual compressive strength of the concrete samples was obtained through destructive tests according to the Taiwan National CNS1232 standard, "Compressive Strength of Concrete Specimens". This standard describes the method that determines the compressive strength of concrete beam samples such as monolithic concrete or coredrilled concrete beam samples. The testing device, an HT-8391 compressing machine, is able to produce up to 200 tons of pressure on the surface of the concrete sample.

Before testing, the surface of the concrete beam was cleaned with a wet tissue to prepare the upper surface of the concrete beam. The location of the core sample on the beams and locations of the RH and NPV tests are shown in Figure 8.

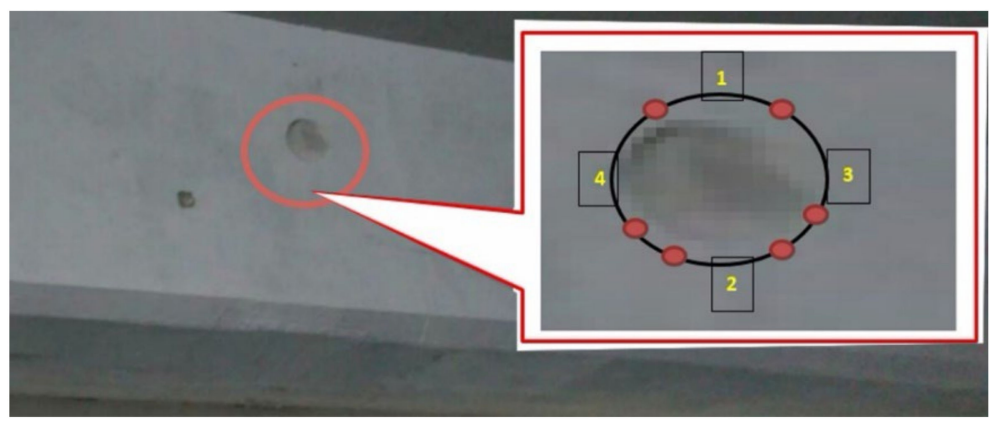

Figure 8. Test locations on the selected test beam.

\subsection{Prediction Model Development \\ 4.3.1. Artificial Neural Networks}

To develop the ANNs model, this paper used Neuro Solutions 7.0 software to construct the neural network models (built-in with EXCEL). A back-propagation network (BPN) was adopted for the concrete compressive strength prediction. Data from a total of 98 samples were collected from the non-structural beams of a basement of a large residential building. Among the data from the 98 samples, 70 of them were randomly chosen as the training dataset for the prediction model. The remaining 28 sample data were set as 
the testing dataset to validate the prediction models. The model input variables were the average rebound number and the average ultrasonic pulse velocity obtained from the in-situ experiments. The model output was the concrete compressive strength. Then, the model training process was used to determine the best prediction model. After the model set up was completed, the training data were loaded to train the ANNs model. The prediction results were compared to the actual concrete compressive strength (obtained from the destructive compressive strength tests on the core samples). The prediction accuracy was evaluated by the root mean square error (RMSE), the Mean Absolute Error (MAE), the Mean Forecast Error (MFE), and the Error to Signal Ratio (ESR) and the mean absolute percentage error (MAPE).

Various modes with different model setups were explored to determine the best-fit model. Previous research has suggested that one or two hidden layers is suitable for most non-linear regression problems. This research tried both one and two hidden layers in the ANNs model setup. Table 2 summarizes the parameters used for the ANNs models developed in this research.

Table 2. Network model parameter settings.

\begin{tabular}{|c|c|c|}
\hline Network Parameters of the Project & \multicolumn{2}{|c|}{ Explanation } \\
\hline Internet usage examples model & \multicolumn{2}{|c|}{ Back-propagation neural network } \\
\hline \multirow{3}{*}{ Sample selection (Exemplars) } & Total Data & 98 \\
\hline & Training Data & 70 \\
\hline & Testing Data & 28 \\
\hline The number (Hidden Layers) & \multicolumn{2}{|c|}{ One Layer and Two Layer } \\
\hline Transfer & \multicolumn{2}{|c|}{ Tanh Axon } \\
\hline Learning Rule & \multicolumn{2}{|c|}{ Levenberg Marqua } \\
\hline Maximum Epochs & \multicolumn{2}{|c|}{ The default value is 200 , and gradually increased } \\
\hline Termination & \multicolumn{2}{|c|}{$\begin{array}{l}\text { 1. The minimum tolerable range (MSE) } \\
\text { 2. The maximum training period (Epochs) }\end{array}$} \\
\hline Cumulative weights update method & \multicolumn{2}{|c|}{ Batch } \\
\hline
\end{tabular}

\subsubsection{Support Vector Machine setting}

This research used the LS-SVM function in MATLAB to develop the support vector machine prediction model. As is the case for ANNs models, the RMSE, MAE, MFE, ESR and MAPE were used to evaluate model performance. The Gaussian Radial Basis Function kernel was adopted in the SVM model, as suggested by the literature. For the RBF kernel SVMs, two parameters, $\sigma$ and $\mathrm{\gamma}$, need to be determined to set up the prediction model.

Similar to the development of the ANNs model, 70 out of the 98 sample data were randomly chosen as the training dataset, and the remaining 28 samples were set as the testing dataset. The model input variables were the average rebound number and the average ultrasonic pulse velocity obtained from the in-situ experiments. The model output was the concrete compressive strength. For RBF kernel SVMs, the sigma indicates the smoothness of the decision surface. The sigma trades off miss-classification of training examples against the simplicity of the decision surface, and a larger sigma indicates stronger smoothing. Gamma is the regularization parameter in the RBF kernel SVMs. A lower gamma minimizes the complexity of the model; a larger gamma creates a better fit of the training data points. The sigma and gamma values are problem specific and must be selected on a trial-and-error basis. RMSE, MAE, MFE, ESR and MAPE were used to evaluate the performance of SVMs prediction models. 


\subsubsection{Adaptive Neural Fuzzy Inference System Setting}

The ANFIS models were developed in MATLAB to train and test the prediction models. Seventy out of the ninety-eight experimental data were set as the training data for the neuron-based fuzzy inference system. Different model setups were explored to determine the best ANFIS prediction model. The remaining 28 samples were set as the testing data. The concrete compressive strengths predicted by the ANFIS models were compared with the actual compressive strength obtained from the destructive test. RMSE, MAE, MFE, ESR and MAPE were used to examine the prediction accuracy. The testing data were used to assess the best ANFIS model from the training process. The development of the ANFIS model in MATLAB followed the steps below:

- Step 1: Import the training and testing data:

$$
\begin{aligned}
& \text { traindata }=x l s r e a d(\text { 'traindata.xlsx') } \\
& \text { testdata }=x l s r e a d\left(' t e s t d a t a . x l s x^{\prime}\right)
\end{aligned}
$$

- Step 2: Create an ANFIS prediction model

In Matlab, "anfisedit" was used to create the ANFIS prediction model. This study tried eight different types of fuzzy membership functions (MFs) in the prediction model development process. These membership functions were triangular MF, trapezoid MF, generalized bell-shaped MF, Gaussian MF, Gaussian combination MF, pi-shaped $\mathrm{MF}$, the difference between two sigmoidal MF, and product of two sigmoidal MF. The researchers also varied the number of membership functions (two, three, four, and five) for the ANFIS model. As result a total of 32 different models were explored during the ANFIS development process. The ANFIS model setup is summarized as follows:

- Number of input membership functions: 2, 3, 4, 5

- MF function type: triangular, trapezoid, generalized bell-shaped, Gaussian, Gaussian combination, pi-shaped, the difference between two sigmoidal, and the product of two sigmoidal

- Output MF type: constant

- FIS training method: hybrid

- $\quad$ Step 3: Set the convergence condition The training model convergence conditions were set to control the model training time. In the beginning, a learning epoch of 10 and the default value of 5000 times was set and then gradually increased. The network error tolerance was set to 0.2 .

- $\quad$ Step 4: Export training results

When the training process was completed, the testing datasets were used to evaluate the training model. The model prediction results were exported to the function below.

$$
\text { Out_value }=\text { evalfis }(\text { testdata, fis1) }
$$

The obtained prediction results were then compared to the actual concrete compression strength to examine the model prediction accuracy. To be consistent, the RMSE, MAE, MFE, ESR and MAPE were used to measure the model prediction performance.

\section{Prediction Results}

\subsection{Research Implication}

The average and standard deviation of the in-situ NDT measurements were calculated for model development and validation. For the RH tests, there were ten measurements taken at each test location. Additionally, for the UPV tests, there were four measurements 
taken at each location. The average and standard deviation of the test samples were calculated using the following equations:

$$
\begin{gathered}
\mathrm{X}_{\mathrm{avg}}=\frac{\sum_{i=1}^{n} x}{n} \\
\mathrm{X}_{\sigma}=\sqrt{\frac{\sum(x-\bar{x})^{2}}{n} .}
\end{gathered}
$$

Among the 98 test samples collected, 70 of them were randomly selected as the training dataset, and 28 were selected as the testing dataset. To obtain the actual concrete compressive strength, the core samples that were taken at each location were brought back to the laboratory for destructive tests. The model prediction accuracy was measured by the root mean square error (RMSE), the mean absolute percentage error (MAPE), the mean absolute error (MAE), the mean forecast error (MFE), and the error to signal ratio (ESR), as illustrated in the following equations:

$$
\begin{array}{r}
\mathrm{RMSE}=\sqrt{\frac{\sum_{i=1}^{n}\left(A_{i}-P_{i}\right)^{2}}{n}} \\
\mathrm{MAPE}=\frac{1}{n} \sum_{i=1}^{n}\left|\frac{A_{i}-P_{i}}{A_{i}}\right| \\
\mathrm{MAE}=\frac{1}{n} \sum_{i=1}^{n}\left|A_{i}-P_{i}\right| \\
\mathrm{MFE}=\frac{1}{n} \sum_{i=1}^{n}\left(P_{i}-A_{i}\right) \\
\mathrm{ESR}=\frac{\frac{1}{n} \sum_{i=1}^{n}\left(A_{i}-P_{i}\right)^{2}}{\frac{1}{n} \sum_{i=1}^{n}\left(P_{i}-\frac{1}{n} \sum_{i=1}^{n} P_{i}\right)^{2}}
\end{array}
$$

where $A_{i}$ is the actual concrete sample compressive strength and $P_{i}$ is the model predicted strength.

In this research, there were three different AI based prediction models: (a) the artificial neural network; (b) the support vector machine; and (c) the adaptive neural fuzzy inference system. For these models, results from the RH and UPV tests were set as the input variables; the concrete compressive strength was set as the output variable. The training datasets were used to train the models. Different model and parameter setups were explored to determine the best setup for each AI method. The training models were then validated by the testing dataset.

\subsection{ANNs Model Prediction Results}

There were two input variables for the ANNs model: the rebound number and the ultrasonic pulse velocity. The output variable was the concrete compressive strength.

- Input 1: the average of $10 \mathrm{RH}$ measurements

- Input 2: the average of 4 UPV measurements

- Output: concrete compressive strength

The functions used in the ANNs were linear regression, a radial basic function network (RBFN) trained by Bayesian regularization, a generalized regression network and multilayer perceptron.

The training error for the ANNs is shown in Table 3. For ANNs model training, generalized regression network produced the best results with a RMSE of 41.97, MAPE of $5.92 \%$, MAE of 80.47, MFE of -50.47 , and ESR of 1.28 . 
Table 3. ANNs training errors.

\begin{tabular}{ccccc}
\hline & $\begin{array}{c}\text { Linear } \\
\text { Regression }\end{array}$ & $\begin{array}{c}\text { RBFN Trained } \\
\text { by Bayesian } \\
\text { Regularization }\end{array}$ & $\begin{array}{c}\text { Generalized } \\
\text { Regression } \\
\text { Network }\end{array}$ & $\begin{array}{c}\text { Multilayer } \\
\text { Perceptron }\end{array}$ \\
\hline RMSE & 76.891911 & 51.986192 & $\mathbf{4 1 . 9 6 5 9 5 2}$ & 75.674336 \\
MAPE(\%) & 15.471931 & 9.266432 & $\mathbf{5 . 9 1 8 1 8 2}$ & 15.086299 \\
MAE & 82.907256 & 102.849302 & $\mathbf{8 0 . 4 7 3 5 8 4}$ & 90.372547 \\
MFE & -57.739648 & -98.372583 & $\mathbf{- 5 0 . 4 7 2 5 4 8}$ & -61.374364 \\
ESR & 3.407829 & 7.287362 & $\mathbf{1 . 2 8 3 6 4 8}$ & 1.297132 \\
\hline
\end{tabular}

Table 3 shows that the generalized regression network model yields the best training results. Next, the testing datasets were used to validate the trained model. The prediction model performances are summarized in Table 4.

Table 4. Prediction results of ANNs models.

\begin{tabular}{ccccc}
\hline & $\begin{array}{c}\text { Linear } \\
\text { Regression }\end{array}$ & $\begin{array}{c}\text { RBFN Trained } \\
\text { by Bayesian } \\
\text { Regularization }\end{array}$ & $\begin{array}{c}\text { Generalized } \\
\text { Regression } \\
\text { Network }\end{array}$ & $\begin{array}{c}\text { Multilayer } \\
\text { Perceptron }\end{array}$ \\
\hline RMSE & $\mathbf{9 5 . 6 5 2 0 1 7}$ & 120.0424 & 107.0982 & 104.68823 \\
MAPE(\%) & $\mathbf{1 4 . 6 9 0 7 2 7}$ & 18.414986 & 14.730913 & 16.449083 \\
MAE & $\mathbf{8 0 . 3 6 8 8 6 5}$ & 106.272740 & 84.247309 & 89.247132 \\
MFE & $\mathbf{- 5 9 . 7 4 2 3 5 7}$ & -102.085052 & -60.145486 & -67.736283 \\
ESR & $\mathbf{3 . 4 1 7 5 4 3}$ & 6.915669 & 4.192067 & 4.137828 \\
\hline
\end{tabular}

In this ANNs simulation, the linear regression function and generalized regression network produced a better performance, with MAPE's of $14.69 \%$ and $14.73 \%$, respectively. The multilayer perceptron model had better RMSE than the generalized regression network; but its MAPE was $2 \%$ higher. The model with the linear regression function produced the best performance compared to models with other functions. The RMSE, MAPE, MAE, MFE, and ESR of the linear regression model were 95.65, 14.6\%, 80.37, -59.74, and 3.42, respectively.

\subsection{SVMs Model Prediction Results}

The SVMs models for this research were developed in the MATLAB environment. As was the case for ANNs models, the input variables for the SVMs models were the average rebound number and the ultrasonic pulse velocity obtained in the in-situ NDT tests. The output variable was the concrete compressive strength. From the literature, there is still no universal method for determining sigma and gamma in the SVMs modeling process. For this research, sigma and gamma were determined by trial and error. The gamma parameter was first fixed at 1000, and then sigma was changed for a different model setup. The SVMs model training results are shown in Table 5 . The SVMs model with best prediction result was the model with a sigma of 32 . Next, the sigma parameter is fixed at 32 , and the gamma was changed for a different model setup. The results are summarized in Table 6.

Table 5. SVMs training errors for two input results with fixed gamma $=1000$.

\begin{tabular}{|c|c|c|c|c|c|c|c|c|c|c|c|}
\hline $\begin{array}{c}\text { Strength } \\
\mathrm{MPa}\end{array}$ & $\begin{array}{l}\text { Sigma } \\
=1\end{array}$ & $\begin{array}{l}\text { Sigma } \\
=2\end{array}$ & $\begin{array}{l}\text { Sigma } \\
=4\end{array}$ & $\begin{array}{l}\text { Sigma } \\
\quad=8\end{array}$ & $\begin{array}{l}\text { Sigma } \\
=16\end{array}$ & $\begin{array}{l}\text { Sigma } \\
=32\end{array}$ & $\begin{array}{l}\text { Sigma } \\
=64\end{array}$ & $\begin{array}{l}\text { Sigma } \\
=128\end{array}$ & $\begin{array}{c}\text { Sigma } \\
=256\end{array}$ & $\begin{array}{l}\text { Sigma } \\
=512\end{array}$ & $\begin{array}{l}\text { Sigma } \\
=1024\end{array}$ \\
\hline RMSE & 225.37 & 238.761 & 127.54 & 71.84 & 67.685 & 70.755 & 74.786 & 76.794 & 79.475 & 84.551 & 88.449 \\
\hline \%МAPE & 34.893 & 38.645 & 18.453 & 10.237 & 10.453 & 10.003 & 10.870 & 10.225 & 11.121 & 12.337 & 12.888 \\
\hline MAE & 175.365 & 188.848 & 104.752 & 59.916 & 56.692 & 55.140 & 56.481 & 59.193 & 62.685 & 67.241 & 71.199 \\
\hline MFE & 110.943 & 89.414 & 35.578 & 8.192 & -7.760 & -15.055 & -14.912 & -14.963 & -10.534 & -8.297 & -4.552 \\
\hline ESR & 0.917 & 0.793 & 0.918 & 0.990 & 0.787 & 0.782 & 0.999 & 1.154 & 1.437 & 1.863 & 2.244 \\
\hline
\end{tabular}


Table 6. SVMs training errors with two input results with fixed sigma $=32$.

\begin{tabular}{ccccc}
\hline $\begin{array}{c}\text { Concrete Strength } \\
\mathbf{M P a} / \mathbf{c m}^{\mathbf{2}}\end{array}$ & Gamma $=\mathbf{1}$ & Gamma $=\mathbf{1 0}$ & Gamma $=\mathbf{1 0 0}$ & Gamma $=\mathbf{1 0 0 0}$ \\
\hline RMSE & 97.980 & 83.296 & 79.233 & $\mathbf{7 0 . 7 5 5}$ \\
\%MAPE & 16.003 & 11.667 & 10.245 & $\mathbf{1 0 . 0 0 3}$ \\
MAE & 85.022 & 66.797 & 58.542 & $\mathbf{5 5 . 1 4 0}$ \\
MFE & -62.248 & -37.794 & -23.801 & $\mathbf{- 1 5 . 0 5 5}$ \\
ESR & 0.795 & 1.935 & 1.127 & $\mathbf{0 . 7 8 2}$ \\
\hline
\end{tabular}

As shown in Table 6, the sigma of 32 and the gamma of 1000 were obtained for the SVMs model with the best training results. Then, the testing dataset was used to validate the best SVMs training model. For the 28 testing samples, prediction model to determine the compressive strengths were compared with the actual compressive strength. The RMSE and MAPE that were obtained were 70.15 and $10.23 \%$, respectively, for the best SVMs training model.

\subsection{ANFIS Model Prediciton Results}

In the MATLAB environment, users can choose different numbers and different types of membership functions in the ANFIS model development process. For this research, ANFIS models with two, three, four, and five membership functions were created to examine the prediction performance of different model setups. In the meantime, eight different types of attribution functions were also explored in the ANFIS models, and the attribute functions were trimf, trapmf, gbellmf, gaussmf, gauss $2 \mathrm{mf}$, pimf, dsigmf, and psigmf.

For this research, a total of 32 different ANFIS models were developed (eight types of attribute function $x$ four types of function number). Among the 32 ANFIS models, the model with the triangular membership function 'trimf' and the model with the trapezoid membership function 'tramf' had the best training results, as shown in Tables 7 and 8 . The average rebound number and average ultrasonic pulse velocity were the ANFIS input variables. The output variable was the concrete compressive strength.

Table 7. Training results of the ANFIS model with triangular membership function 'trimf'.

\begin{tabular}{ccccc}
\hline Concrete Strength (MPa) & $\mathbf{2 ~ 2}$ & $\mathbf{3 3}$ & $\mathbf{4 4}$ & $\mathbf{5 5}$ \\
\hline RMSE & 94.997 & $\mathbf{6 5 . 7 6 5}$ & $2.98 \times 10^{3}$ & 390.926 \\
MAPE (\%) & 14.000 & $\mathbf{9 . 9 9 4}$ & 126.478 & 50.085 \\
MAE & 76.887 & $\mathbf{5 2 . 9 6 5}$ & 720.754 & 177.836 \\
MFE & -53.776 & $\mathbf{- 2 . 8 2 7}$ & 587.964 & -17.507 \\
ESR & 2.904 & $\mathbf{0 . 7 9 7}$ & 1.028 & 0.799 \\
\hline
\end{tabular}

Table 8. Training error results of the ANFIS model with the trapezoid membership function 'tramf'.

\begin{tabular}{ccccc}
\hline Concrete Strength (MPa) & $\mathbf{2 ~ 2}$ & $\mathbf{3 ~ 3}$ & $\mathbf{4 4}$ & $\mathbf{5 5}$ \\
\hline RMSE & 115.004 & $\mathbf{9 4 . 0 0 1}$ & 460.393 & 271.861 \\
MAPE (\%) & 16.993 & $\mathbf{1 3 . 0 4 6}$ & 47.993 & 35.937 \\
MAE & 95.973 & $\mathbf{6 5 . 2 3 7}$ & 276.482 & 168.496 \\
MFE & -77.827 & $\mathbf{1 1 . 3 6 2}$ & -164.447 & -25.646 \\
ESR & 4.436 & $\mathbf{1 . 0 2 0}$ & 1.184 & 0.759 \\
\hline
\end{tabular}

After obtaining the best ANFIS training models, the validation was conducted by introducing the testing data to examine the model prediction accuracy. The ANFIS model prediction results are summarized in Tables 9 and 10. 
Table 9. Prediction results of the ANFIS model with the triangular membership function 'trimf'.

\begin{tabular}{ccccc}
\hline Concrete Strength (MPa) & $\mathbf{2 ~ 2}$ & $\mathbf{3 ~ 3}$ & $\mathbf{4 4}$ & $\mathbf{5 5}$ \\
\hline RMSE & 95.221 & $\mathbf{6 4 . 4 8 9}$ & $3.21 \times 10^{3}$ & 393.210 \\
MAPE (\%) & 14.086 & $\mathbf{1 0 . 0 1 9}$ & 126.344 & 49.439 \\
MAE & 77.107 & $\mathbf{5 3 . 0 2 5}$ & 721.955 & 180.336 \\
MFE & -52.932 & $\mathbf{- 2 . 9 7 3}$ & 585.836 & -17.787 \\
ESR & 3.034 & $\mathbf{0 . 7 8 7}$ & 0.992 & 0.826 \\
\hline
\end{tabular}

Table 10. Prediction results of the ANFIS model with the trapezoid membership function 'tramf'.

\begin{tabular}{ccccc}
\hline Concrete Strength (MPa) & $\mathbf{2 ~ 2}$ & $\mathbf{3 ~ 3}$ & $\mathbf{4 4}$ & $\mathbf{5 5}$ \\
\hline RMSE & 114.845 & $\mathbf{9 4 . 1 5 0}$ & 456.313 & 272.701 \\
MAPE (\%) & 17.135 & $\mathbf{1 2 . 9 2 4}$ & 48.944 & 36.643 \\
MAE & 96.353 & $\mathbf{6 4 . 2 0 8}$ & 275.324 & 169.001 \\
MFE & -75.985 & $\mathbf{1 2 . 9 6 3}$ & -160.186 & -26.294 \\
ESR & 4.298 & $\mathbf{0 . 9 9 3}$ & 1.086 & 0.782 \\
\hline
\end{tabular}

The models with the best prediction performance are summarized in Table 11. All three AI methods have a MAPE under $15 \%$, while the ANFIS model has the best prediction results, with a MAPE of $10.01 \%$. For the ANNs models, the linear regression model has the best prediction results. Table 12 shows a portion of the weights and the bias of the best ANNs prediction model. For the SVMs models, the RBF kernel SVMs with a sigma of 32 and a gamma of 1000 yields the best prediction results. For the ANFIS models, the model with two triangular membership functions for each input produces the best prediction results. Overall, the ANFIS models yield the best prediction performance, with an RMSE of 64.49 and a MAPE of $10.01 \%$ when validated using the randomly chosen testing dataset. Moreover, both the prediction results of the SVMs and ANFIS models outperform the ANNs model, which is the model that is the most commonly used by other researchers.

Table 11. Best model prediction accuracy summary.

\begin{tabular}{ccccccc}
\hline $\begin{array}{c}\text { Forecasting } \\
\text { Model }\end{array}$ & Parameter Setting & RMSE & MAPE (\%) & MAE & MFE & ESR \\
\hline ANNs & linear regression & 95.65 & 14.69 & 80.37 & -59.74 & 3.42 \\
\hline SVMs & gamma = 1000, sigma $=32$ & 70.76 & 10.00 & 55.14 & -15.06 & 0.78 \\
\hline ANFIS & Two triangular membership & 64.49 & 10.01 & 53.03 & -2.97 & 0.79 \\
\hline
\end{tabular}

Table 12. Weights and bias of the best ANNs model.

\begin{tabular}{|c|c|c|c|c|c|c|c|c|c|c|c|c|}
\hline & Weights & $\mathrm{W}_{1 \mathrm{i}}$ & $\mathrm{W}_{2 \mathrm{i}}$ & $\mathbf{W}_{3 \mathrm{i}}$ & $\mathrm{W}_{4 \mathrm{i}}$ & $W_{5 i}$ & $W_{6 i}$ & $\mathbf{w}_{7 \mathrm{i}}$ & $\mathrm{W}_{8 \mathrm{i}}$ & $\mathrm{W}_{9 \mathrm{i}}$ & $\mathrm{W}_{10 \mathrm{i}}$ & Bias \\
\hline$W_{1}(1 \times 10)$ & Layer 1 & -0.445 & 0.563 & 1.456 & -2.159 & 0.849 & 0.849 & 0.849 & 0.849 & 0.849 & 0.717 & \\
\hline$W_{2, \text { col1 }}(10 \times 7)$ & Layer 2 & 0.261 & -2.314 & 1.347 & -1.352 & -0.013 & -0.013 & -0.013 & -0.013 & -0.013 & 0.657 & 0.921 \\
\hline$W_{O}(7 \times 1)$ & & -0.329 & 1.020 & 0.598 & 0.230 & -1.239 & 1.254 & 0.962 & & & & 0.487 \\
\hline
\end{tabular}

\section{Conclusions}

To improve the estimation results of in-situ non-destructive concrete compressive strength tests, artificial intelligence methods were applied to analyze the experimental data from the rebound hammer test and the ultrasonic pulse velocity test. Three artificial intelligence techniques (artificial neural networks, support vector machines, and adaptive neural fuzzy inference system) were adopted to develop concrete compressive strength prediction models. In-situ experimental data form a total of 98 samples were collected from the non-structural beams of a large residential complex. Both RH and UPV tests were 
conducted on the designated test locations, and core samples were then taken to obtain the actual concrete compressive strength at each test location. Among the 98 samples, 70 samples were randomly chosen to be the training data in this study. The remaining 28 samples were set as the testing data to evaluate the model prediction accuracy. When applying the three AI methods, different model setups were explored in the model development process. The two model input variables were the average of the rebound hammer number and the average of the ultrasonic pulse velocity. The model output was the concrete compressive strength. During the model development process, the 70 testing data were used to develop and train the models. The training models with the best results were identified and were then validated using the 28 testing data.

From the literature, it has been shown that traditional concrete compressive strength estimations have a mean absolute percentage error (MAPE) of over $20 \%$ when compared to the actual compressive strength obtained by destructive tests. Moreover, most of the previous research used samples from a laboratory instead of samples from the actual structure. For this research, in-situ non-destructive concrete compressive strength tests were conducted, and core samples were taken to identify the actual concrete compressive strength. By applying the AI techniques to the data analysis, the research results show that the ANNs, SVMs, and ANFIS prediction models yield satisfactory concrete strength prediction results with MAPE's of $14.69 \%, 10.23 \%$, and $10.01 \%$, respectively. This shows a significant improvement compared to the results from previous research. The research results provide a valuable reference for both researchers and the industry practitioners when evaluating in-situ concrete compressive strength using NDT methods.

Author Contributions: T.Q.L.N. developed models and analyzed the data. D.-L.C. conducted the experiment. Y.-R.W. initiated the original research ideas. Y.-R.W. supervised the experiments and data analysis. T.Q.L.N. took the lead in writing the manuscript with help from Y.-R.W. and D.-L.C. All authors have read and agreed to the published version of the manuscript.

Funding: This material is based upon work supported by the Ministry of Science and Technology, TAIWAN under grant no. MOST 107-2221-E-992-050.

Informed Consent Statement: Written informed consent was obtained from the individual(s) for the publication of any potentially identifiable images or data included in this article.

Data Availability Statement: The research data were collected in collaboration with a material testing laboratory in Taiwan. The testing samples were provided by the laboratory's customer. The authors of this research have signed a non-disclosure agreement to keep the raw data information confidential. The authors can only reveal certain information (such as analytical results) according to this agreement. For more information concerning the experimental data, the corresponding author can be reached at yrwang@nkust.edu.tw.

Conflicts of Interest: The authors declare no conflict of interest.

\section{References}

1. Zhang, J.; Haghighat, F. Development of Artificial Neural Network based heat convection algorithm for thermal simulation of large rectangular cross-sectional area Earth-to-Air Heat Exchangers. Energy Build. 2010, 42, 435-440. [CrossRef]

2. Olofsson, T.; Andersson, S.; Östin, R. A method for predicting the annual building heating demand based on limited performance data. Energy Build. 1998, 28, 101-108. [CrossRef]

3. Kalogirou, S.A.; Bojic, M. Artificial neural networks for the prediction of the energy consumption of a passive solar building. Energy 2000, 25, 479-491. [CrossRef]

4. Hou, Z.; Lian, Z.; Yao, Y.; Yuan, X. Cooling-load prediction by the combination of rough set theory and an artificial neural-network based on data-fusion technique. Appl. Energy 2006, 83, 1033-1046. [CrossRef]

5. Ben-Nakhi, A.E.; Mahmoud, M.A. Cooling load prediction for buildings using general regression neural networks. Energy Convers. Manag. 2004, 45, 2127-2141. [CrossRef]

6. Bungey, J.H.; Millard, J.H.; Grantham, M.G. Testing of Concrete in Structures; Taylor and Francis: New York, NY, USA, 2006 ; p. 352.

7. Available online: http://www.engineeringcivil.com/rebound-hammer-test.html (accessed on 30 July 2021).

8. Bhat, S.T.; Lovell, C.W. Use of Coal Combustion Residues and Foundry Sands in Flowable Fill; Joint Highway Research Project, Indiana Department of Transportation and Purdue University: West Lafayette, IN, USA, 1996.

9. ACI-229R. Controlled-Low Strength Materials (Reproved 2005); American Concrete Institute: Farmington Hills, MI, USA, 2005. 
10. Komloš, K.; Popovics, S.; Nürnbergerová, T.; Babál, B. Ultrasonic pulse velocity test of concrete properties as specified in various standards. Cem. Concr. Compos. 1996, 18, 357-364. [CrossRef]

11. Khademi, F.; Jamal, S.M. Predicting the 28 Days Compressive Strength of Concrete Using Artificial Neural Network. $i-M a n a g . ~ J$. Civ. Eng. 2016, 6, 34-47.

12. Nikoo, M.; Zarfam, P.; Nikoo, M. Determining Displacement in Concrete Reinforcement Building with Using Evolutionary Artificial Neural Networks. World Appl. Sci. J. 2012, 16, 1699-1708.

13. Kahraman, S. Evaluation of simple methods for assessing the uniaxial compressive strength of rock. Int. J. Rock Mech. Min. Sci. 2001, 38, 981-994. [CrossRef]

14. Qasrawi, H.Y. Concrete strength by combined nondestructive methods simply and reliably predicted. Cem. Concr. Res. 2000, 30, 739-746. [CrossRef]

15. Nehdi, M.; Chabib, H.E.; Naggar, A. Predicting performance of self-compacting concrete mixtures using artificial neural networks. ACI Mater. J. 2001, 98, 394-401.

16. Trtnink, G.; Kavcic, B.; Turk, G. Predicting of concrete strength using ultrasonic pulse velocity and artificial neural networks. Ultrasonics 2009, 49, 53-60. [CrossRef] [PubMed]

17. Malek, J.; Kaouther, M. Destructive and Non-destructive Testing of Concrete Structures. Jordan J. Civ. Eng. $2014,8,432-447$.

18. Available online: http:/ / www.engineeringcivil.com/upv-test.html (accessed on 30 July 2021).

19. Huang, Q.; Gardoni, P.; Hurlebaus, S. Predicting Concrete Compressive Strength Using Ultrasonic Pulse Velocity and Rebound Number. ACI Mater. J. 2001, 108, 403-412.

20. Basu, A.; Aydin, A. A method for normalization of Schmidt hammer rebound values. Int. J. Rock Mech. Min. Sci. 2004, 41, 1211-1214. [CrossRef]

21. ASTM C805-02. Standard Test Method for Rebound Number of Hardened Concrete; ASTM International: West Conshohocken, PA USA, 2002.

22. Yusuf, I.T.; Jimoh, Y.A.; Salami, W.A. An appropriate relationship between flexural strength and compressive strength of palm kernel shell concrete. Alex. Eng. J. 2016, 55, 1553-1562. [CrossRef]

23. Naik, T.R.; Malhotra, V.M.; Popovics, J.S. The ultrasonic pulse velocity method. In Handbook on Nondestructive Testing of Concrete; Malhotra, V.M., Carino, N.J., Eds.; CRC Press: New York, NY, USA, 2004.

24. American Concrete Institute (ACI 228). Nondestructive Test Methods for Evaluation of Concrete in Structures (ACI 228.2R-98); American Concrete Institute: Farmington Hills, MI, USA, 1998.

25. Kou, S.-C.; Poon, C.S.; Wan, H.-W. Properties of concrete prepared with low-grade recycled aggregates. Constr. Build. Mater. 2012, 36, 881-889. [CrossRef]

26. Al-Mufti, R.L.; Fried, A. The early age non-destructive testing of concrete made with recycled concrete aggregate. Constr. Build. Mater. 2012, 37, 379-386. [CrossRef]

27. Barluenga, G.; Puentes, J.; Palomar, I. Early age monitoring of self-compacting concrete with mineral additions. Constr. Build. Mater. 2015, 77, 66-73. [CrossRef]

28. Molero, M.; Segura, I.; Izquierdo, M.; Fuente, J.; Anaya, J. Sand/cement ratio evaluation on mortar using neural networks and ultrasonic transmission inspection. Ultrasonics 2009, 49, 231-237. [CrossRef]

29. Naffa, S.O.; Goueygou, M.; Piwakowski, B.; Buyle-Bodin, F. Detection of chemical damage in concrete using ultrasound. Ultrasonics 2002, 40, 247-251. [CrossRef]

30. Ohtsu, M.; Shigeishi, M.; Sakata, Y. Nondestructive evaluation of defects in concrete by quantitative acoustic emission and ultrasonics. Ultrasonics 1998, 36, 187-195. [CrossRef]

31. Bogas, J.A.; Gomes, M.D.G.; Gomes, A. Compressive strength evaluation of structural lightweight concrete by non-destructive ultrasonic pulse velocity method. Ultrasonics 2013, 53, 962-972. [CrossRef] [PubMed]

32. Sztukiewicz, R.J. Application of ultrasonic methods in asphalt concrete testing. Ultrasonics 1991, 29, 5-12. [CrossRef]

33. Colombo, M.; Felicetti, R. New NDT techniques for the assessment of fire-damaged concrete structures. Fire Saf. J. 2007, 42, 461-472. [CrossRef]

34. BSI. Method for Determination of Compressive Strength of Concrete Cubes; British Standards Institution: London, UK, 1983.

35. ASTM C597-83. Standard Test Method for Pulse Velocity through Concrete; ASTM International: West Conshohocken, PA, USA, 1991.

36. Marfisi, E.; Burgoyne, C.; Amin, M.; Hall, L. The use of MRI to observe the structure of concrete. Mag. Concr. Res. 2005, 57, 101-109. [CrossRef]

37. Prassianakis, I.; Giokas, P. Mechanical properties of old concrete using destructive and ultrasonic non-destructive testing methods. Mag. Concr. Res. 2003, 55, 171-176. [CrossRef]

38. Trtnik, G.; Turk, G. Influence of superplasticizers on the evolution of ultrasonic P-wave velocity through cement pastes at early age. Cem. Concr. Res. 2013, 51, 22-31. [CrossRef]

39. Vipulanandan, C.; Garas, V. Electrical resistivity, pulse velocity, and compressive properties of carbon fiber-reinforced cement mortar. J. Mater. Civ. Eng. 2008, 20, 93-101. [CrossRef]

40. Selcuk, L.; Nar, A. Prediction of Uniaxial Compressive Strength of Intact Rocks Using Ultrasonic Pulse Velocity and Rebound Hammer Number. J. Eng. Geol. Hydrogeol. 2015, 3, 67-75. [CrossRef]

41. Ulucan, Z.Ç.; Türk, K.; Karataş, M. Effect of mineral admixtures on the correlation between ultrasonic velocity and compressive strength for self-compacting concrete. Russ. J. Nondestruct. Test. 2008, 44, 367-374. [CrossRef] 
42. Mohammed, B.S.; Azmi, N.J.; Abdullahi, M. Evaluation of rubbercrete based on ultrasonic pulse velocity and rebound hammer tests. Constr. Build. Mater. 2011, 25, 1388-1397. [CrossRef]

43. Ikpong, A. The relationship between the strength and non-destructive parameters of rice husk ash concrete. Cem. Concr. Res. 1993, 23, 387-398. [CrossRef]

44. Islam, S.M.; Hashim, R.; Islam, A.B.M.S.; Kurnia, R. Effect of Peat on Physicomechanical Properties of Cemented Brick. Sci. World J. 2014, 2014, 328516. [CrossRef] [PubMed]

45. Asteris, P.G.; Mokos, V.G. Concrete compressive strength using artificial neural networks. Neural Comput. Appl. 2019, 32, 11807-11826. [CrossRef]

46. Pazouki, G.; Pourghorban, A. Using a hybrid artificial intelligence method for estimating the compressive strength of recycled aggregate self-compacting concrete. Eur. J. Environ. Civ. Eng. 2021, 8, 20-34. [CrossRef]

47. Bonagura, M.; Nobile, L. Artificial Neural Network (ANN) Approach for Predicting Concrete Compressive Strength by SonReb. Struct. Durab. Health Monit. 2021, 15, 125-137. [CrossRef]

48. Whitehurst, E.A. Soniscope tests concrete structures. J. Proc. 1951, 47, 433-444.

49. Jaksa, M.B.; Maier, H.R. Future challenges for artificial neural network modeling in geotechnical engineering. In Proceedings of the 12th International Conference of International Association for Computer Methods and Advances in Geomechanics (IACMAG), Goa, India, 1-6 October 2008.

50. Elhag, T.M.S.; Boussabaine, A.H. Tender price estimation using artificial neural networks II: Modelling. J. Financ. Manag. Prop. Constr. 2002, 7, 49-64.

51. Elhag, T.M.S. Cost modeling: Neural networks vs. regression techniques. In Proceedings of the International Conference on Construction Information Technology (INCITE), Construction Industry Development Board Malaysia (CIDB), Langkawi, Malaysia, 18-21 February 2004.

52. Haykin, S. Neural Networks, a Comprehensive Foundation, 2nd ed.; Prentice Hall: Denver, CO, USA, 1999.

53. Shah, A.A.; Alsayed, S.H.; Abbas, H.; Al-Salloum, Y.A. Predicting residual strength of non-linear ultrasonically evaluated damaged concrete using artificial neural network. Constr. Build. Mater. 2012, 29, 42-50. [CrossRef]

54. Sazli, M.H. A brief review of feed-forward neural networks. Commun. Fac. Sci. Univ. Ank. 2006, 11-17. [CrossRef]

55. Samarasinghe, S. Neural Networks for Applied Sciences and Engineering; Auerbach Publications: London, UK, 2007.

56. Wu, Y.; Wang, H.; Zhang, B.; Du, K.-L. Using Radial Basis Function Networks for Function Approximation and Classification. ISRN Appl. Math. 2012, 2012, 324194. [CrossRef]

57. Khademi, F.; Behfarnia, K. Evaluation of concrete compressive strength using artificial neural network and multiple linear regression models. Iran Univ. Sci. Technol. 2016, 6, 423-432.

58. Chou, J.-S.; Bui, D.K. Modeling heating and cooling loads by artificial intelligence for energy-efficient building design. Energy Build. 2014, 82, 437-446. [CrossRef]

59. IS 13311: Standard Code of Practice for Non-Destructive Testing of Concrete: Part 1-Ultrasonic Pulse Velocity; Bureau of Indian Standards: New Delhi, India, 1992.

60. Liong, S.-Y.; Sivapragasam, C. Flood Stage Forecasting with Support Vector Machines. JAWRA J. Am. Water Resour. Assoc. 2002, 38, 173-186. [CrossRef]

61. He, J.; Hu, H.-J.; Harrison, R.; Tai, P.C.; Pan, Y. Transmembrane segments prediction and understanding using support vector machine and decision tree. Expert Syst. Appl. 2006, 30, 64-72. [CrossRef]

62. Fletcher, R.; Johnson, T. On the Stability of Null-Space Methods for KKT Systems. SIAM J. Matrix Anal. Appl. 1997, 18, 938-958. [CrossRef]

63. Sun, J.-G. Structured backward errors for KKT systems. Linear Algebra Its Appl. 1999, 288, 75-88. [CrossRef]

64. Golub, G.H.; Van Loan, C.F. Matrix Computations; John Hopkins University Press: Baltimore, MD, USA, 1989.

65. Available online: https://www.researchgate.net/profile/...it_is...ANFIS/.../anfis (accessed on 30 July 2021).

66. Quej, V.H.; Almorox, J.; Arnaldo, J.A.; Saito, L. ANFIS, SVM and ANN soft-computing techniques to estimate daily global solar radiation in a warm sub-humid environment. J. Atmos. Sol.-Terr. Phys. 2017, 155, 62-70. [CrossRef]

67. Tharmaratnam, K.; Tan, B. Attenuation of ultrasonic pulse in cement mortar. Cem. Concr. Res. 1990, 20, 335-345. [CrossRef] 